\title{
Free-Flight Terrestrial Rocket Lander Demonstration for NASA's Autonomous Landing and Hazard Avoidance Technology (ALHAT) System
}

\author{
David K. Rutishauser ${ }^{*}$ Chirold D. Epp, ${ }^{\dagger}$ and Edward A. Robertson ${ }^{*}$ \\ NASA Johnson Space Center, Houston, Texas, 77058
}

The Autonomous Landing Hazard Avoidance Technology (ALHAT) Project is chartered to develop and mature to a Technology Readiness Level (TRL) of six an autonomous system combining guidance, navigation and control with terrain sensing and recognition functions for crewed, cargo, and robotic planetary landing vehicles. The ALHAT System must be capable of identifying and avoiding surface hazards to enable a safe and accurate landing to within tens of meters of designated and certified landing sites anywhere on a planetary surface under any lighting conditions. Since its inception in 2006, the ALHAT Project has executed four field test campaigns to characterize and mature sensors and algorithms that support real-time hazard detection and global/local precision navigation for planetary landings. The driving objective for Government Fiscal Year 2012 (GFY2012) is to successfully demonstrate autonomous, real-time, closed loop operation of the ALHAT system in a realistic free flight scenario on Earth using the Morpheus lander developed at the Johnson Space Center (JSC). This goal represents an aggressive target consistent with a lean engineering culture of rapid prototyping and development. This culture is characterized by prioritizing early implementation to gain practical lessons learned and then building on this knowledge with subsequent prototyping design cycles of increasing complexity culminating in the implementation of the baseline design. This paper provides an overview of the ALHAT/Morpheus flight demonstration activities in GFY2012, including accomplishments, current status, results, and lessons learned. The ALHAT/Morpheus effort is also described in the context of a technology path in support of future crewed and robotic planetary exploration missions based upon the core sensing functions of the ALHAT system: Terrain Relative Navigation (TRN), Hazard Detection and Avoidance (HDA), and Hazard Relative Navigation (HRN).

$\begin{array}{ll} & \\ A L H A T & =\text { Autonomous Landing and Hazard Avoidance Technology } \\ A P U & =\text { Avionics and Power Unit } \\ D E M & =\text { Digital Elevation Map } \\ G F Y & =\text { Government Fiscal Year } \\ G N \& C & =\text { Guidance, Navigation, and Control } \\ H D A & =\text { Hazard Detection and Avoidance } \\ H D P & =\text { Hazard Detection Phase } \\ H D S & =\text { Hazard Detection System } \\ H R N & =\text { Hazard Relative Navigation } \\ I L P & =\text { Intended Landing Point } \\ I M U & =\text { Inertial Measurement Unit } \\ L D T R & =\text { Long Distance Test Range } \\ L I D A R & =\text { Light Detection and Ranging } \\ P P S & =\text { Pulse Per Second } \\ T R L & =\text { Technical Readiness Level } \\ T R N & =\text { Terrain Relative Navigation } \\ V T B & =\text { Vertical Testbed }\end{array}$

\footnotetext{
* Systems Engineering and Integration Lead, ALHAT Project, Aeroscience and Flight Mechanics Division, 2101 NASA Parkway/EG6

${ }^{\dagger}$ Manager, ALHAT Project, Aeroscience and Flight Mechanics Division, 2101 NASA Parkway/EG1, AIAA member

* Deputy Manager, ALHAT Project, Aeroscience and Flight Mechanics Division, 2101 NASA Parkway/EG5
}

1

American Institute of Aeronautics and Astronautics 


\section{Introduction}

A $\mathrm{n}$ examination of the challenges experienced during the Apollo program lunar landings substantiates the need for advanced technologies to identify safe landing locations during planetary powered descent, and precisely guide a vehicle to the selected location. ${ }^{1}$ Since 2006, NASA's Autonomous Landing and Hazard Avoidance Technology (ALHAT) project has advanced the state-of-the-art in technologies and techniques for hazard detection and precision landing that are applicable to crewed, cargo, or robotic planetary landing missions. ${ }^{2,3}$ ALHAT technologies have been validated in numerous field test campaigns using airplanes, helicopters, and rocket-powered Vertical TestBeds (VTBs). ${ }^{4,5}$ As of the writing of this paper, a closed-loop, real-time, Technical Readiness Level $(T R L)^{6}$ six demonstration of an integrated ALHAT implementation on a rocket-powered VTB has yet to be accomplished. An ALHAT system has been integrated with the NASA Johnson Space Center's Morpheus lander ${ }^{7}$ and is currently being tested in preparation for such a demonstration. The ALHAT and Morpheus projects were tasked with this goal for Government Fiscal Year 2012 (GFY2012). This goal is consistent with NASA's Space Technology Roadmap that defines the capabilities required for human and robotic space exploration. ${ }^{8}$ This paper provides an overview of the ALHAT project status, activities, lessons learned, and progress towards a terrestrial TRL six demonstration.

\section{Background and Related Work}

The thread of interest and priority allocated to the topic of autonomous hazard detection and avoidance for planetary landings can be traced through the duration of human space exploration. Early in the Apollo Program an investigation of remote hazard detection for an automatic Lunar Excursion Module (LEM) concept was explored. ${ }^{9}$ This early technique was not autonomous, instead using an astronaut observer in the Crew Service Module. Twentyfive years later, landing hazard avoidance continued to be recognized as a technology requirement for robotic solar system exploration, ${ }^{10}$ and technology development to support autonomous hazard detection and avoidance took shape in this time period. ${ }^{11}$ More recently in 2005, with NASA's human exploration goals focused on a return to the Moon by 2020, NASA Headquarters chartered the ALHAT Project to develop autonomous precision landing and hazard avoidance technologies to a TRL of six. ${ }^{12}$ In a similar timeframe, a parallel effort in Europe was initiated in the Aurora Core Programme, providing a foundation for the European Space Agencies' current goal of a robotic polar lunar mission in 2018. ${ }^{13}$ ALHAT technologies have also been in development in Canada, the United Kingdom, and China. ${ }^{14,15,16}$ Commercial entities have invested internal research and development funds to investigate algorithms for hazard detection and for development and field-testing of hazard detection and avoidance systems. ${ }^{17,18}$ This overview is not intended to be a comprehensive list of ALHAT related efforts, but to underscore the long-standing global investment in autonomous hazard detection, avoidance, and precision landing capabilities.

Providing the relevant environment for an ALHAT TRL six demonstration is the Morpheus lander. Morpheus is a prototype robotic planetary lander that serves as a VTB for advanced spacecraft technologies. The two key technologies that Morpheus is currently testing are LOX/Methane propulsion and ALHAT. In the fall of 2011, the ALHAT and Morpheus projects were tasked with completing a TRL six integrated demonstration by the end of GFY2012. This aggressive GFY2012 goal is consistent with a lean/agile engineering development approach which prioritizes early prototype development and testing early and often to drive out design issues, operational concepts, and requirements. ${ }^{19}$ At that point in time, however, key ALHAT components were still being assembled, significant software functions had not yet been written, and Morpheus was in the midst of a major upgrade and had yet to demonstrate free-flight capability. The next section provides an overview of ALHAT technologies and a status of their level of maturity as of the beginning of GFY2012.

\section{Technology Overview}

In addition to ALHAT representing an integrated solution for a particular mission, ALHAT is also a portfolio of key exploration technologies and techniques that can be tailored for a variety of mission requirements. The ALHAT project has advanced the state-of-the-art in all its constituent systems and methods, and the overview provided in this section provides context for the description of recent and ongoing test activities in subsequent sections. 


\section{A. Techniques}

An ALHAT system uses three modes of operation during a planetary powered descent: 1) Terrain Relative Navigation (TRN), 2) Hazard Detection and Avoidance (HDA), and 3) Hazard Relative Navigation (HRN). TRN compares sensor data with onboard map data to provide precision global navigation. HDA scans the intended landing area and determines safe landing locations. HRN provides precision local navigation to the chosen safe site using nearby surface features. Figure 1 shows where these operational modes are performed in a representative lunar descent and landing trajectory profile. Also annotated in the figure is the portion of the powered descent that will be flown in the VTB tests planned for late GFY2012.

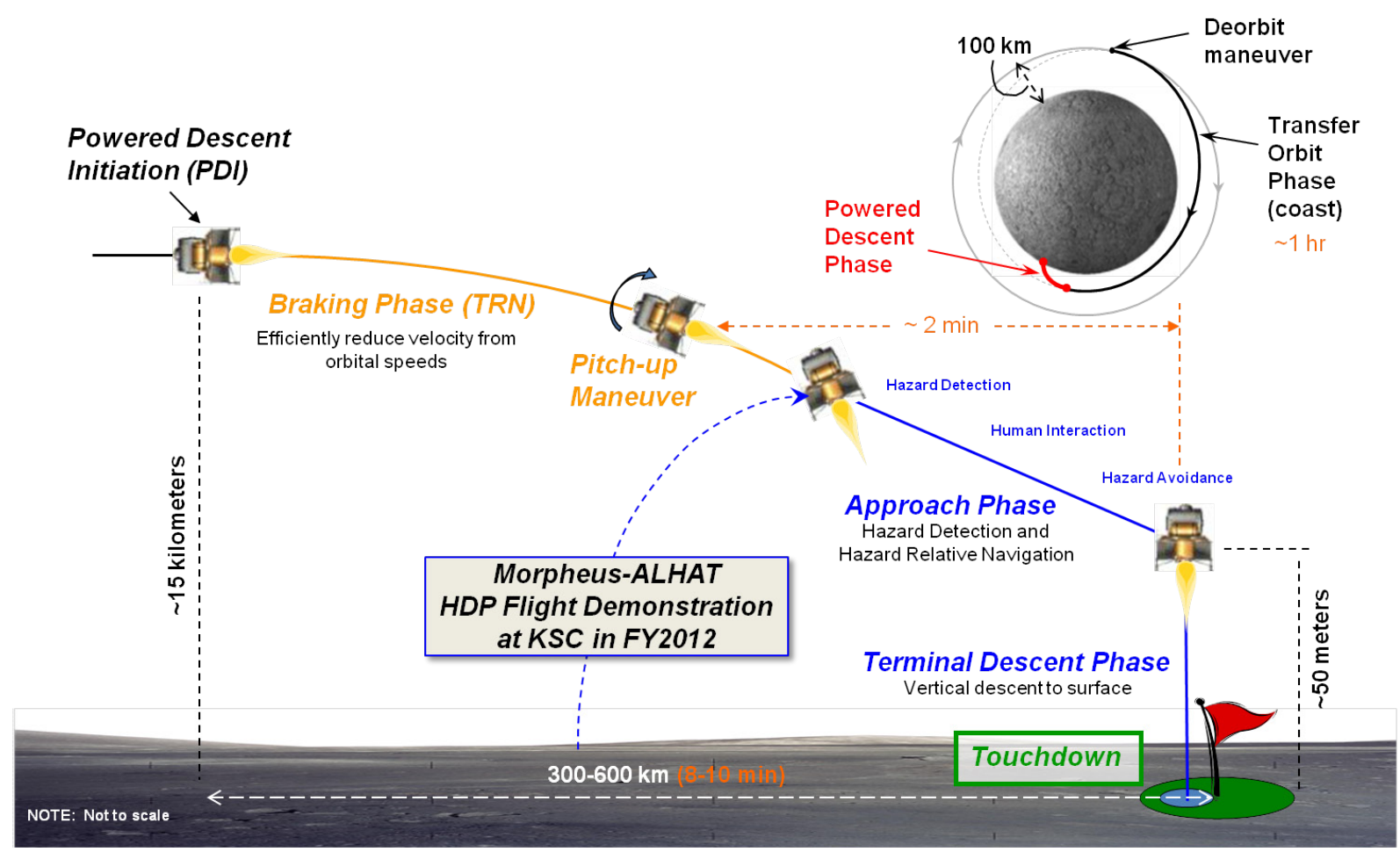

Figure 1. Example powered descent profile with ALHAT functional modes.

The ALHAT project conducted four field tests to characterize the performance of TRN, HDA, and HRN. ${ }^{3}$ Tests of TRN algorithms paired appropriately with optical camera, flash Light Detection and Ranging (LIDAR), and laser altimeter sensors flown over natural lunar-like and Mars-like terrains in an airplane showed that all configurations were able to meet the project's $90 \mathrm{~m} 3$-sigma landing accuracy requirement. The LIDAR configuration specifically is considered verified to a TRL level of 4. TRN testing is not part of the GFY2012 test campaign and will not be discussed further in this paper. The HDA and HRN functions are discussed in the next section.

\section{B. Algorithms and Processing}

Hazard detection in an ALHAT system must produce a solution in about 10 seconds for a typical powered descent. ${ }^{\S}$ This processing window includes scanning an area about the Intended Landing Point (ILP), producing a DEM from the mosaic scan data, and determining the safe sites. The initial algorithm tested in ALHAT Field Test 1 does not meet this computation time requirement on a single processor. However, the algorithm was verified to meet the requirement when parallelized and executed on a multi-core processor. ${ }^{20}$ Although by the end of Field Test 1 the HDA and HRN algorithms were considered to be at TRL levels 5 and 4, respectively, the algorithms used in the current ALHAT implementation have evolved considerably from this initial version, and are computationally more demanding. ${ }^{21}$ The parallelized implementation of the new algorithms was also open work at the beginning of GFY2012. A TRL 6 demonstration of these new algorithms will therefore represent substantial progress in ALHAT processing technologies.

$\S$ This is an internal project requirement primarily based on conserving propellant, protecting for a significant divert, and providing roughly 25 seconds for the crew to evaluate landing options and select a new ILP.

3

American Institute of Aeronautics and Astronautics 


\section{Sensors}

The ALHAT project has developed state-of-the-art sensor technologies to support TRN, HDA, and HRN. The ALHAT Doppler LIDAR is capable of providing ground relative velocity vectors, ground relative range, and vehicle attitude as navigation inputs to a vehicle GN\&C system. ${ }^{22}$ The Doppler LIDAR is considered to be at a TRL level of 4 from testing a breadboard version in ALHAT Field Test 2. ${ }^{3}$ The ALHAT Laser Altimeter has a much greater operational range than the Doppler LIDAR, and is an alternate sensor to the flash LIDAR for the TRN

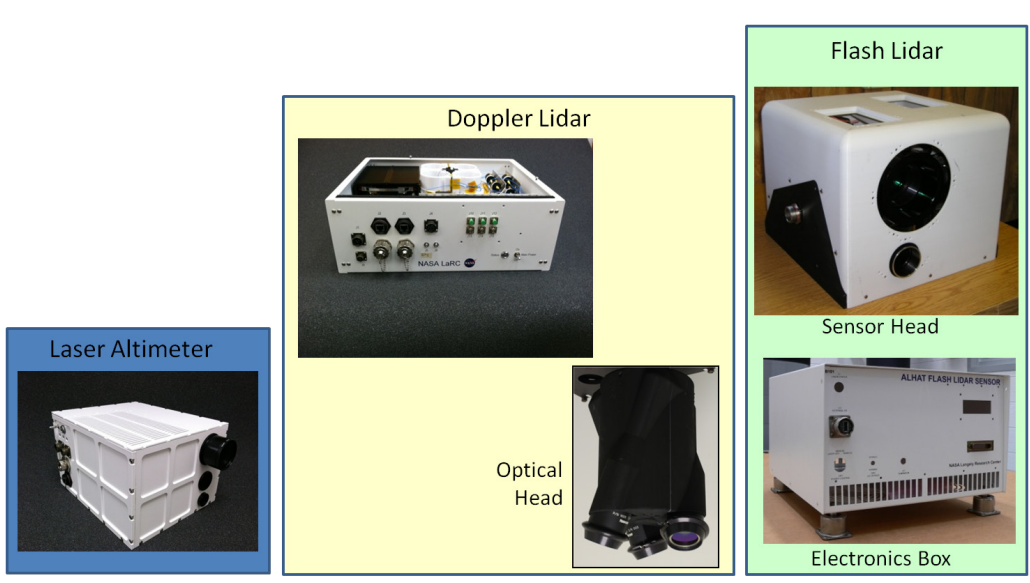

Figure 2. ALHAT sensor subsystems.

function. The Laser Altimeter has been evaluated in ALHAT Field Test 4 and shown to have $8 \mathrm{~cm}$ precision at 30 $\mathrm{km}$ range, exceeding the ALHAT requirements for TRN. ${ }^{23}$ The ALHAT Flash LIDAR system is the primary sensor for the HDA and HRN functions. Its performance was evaluated in the context of these functions in ALHAT Field Tests 1 and 4, and, most recently, during ground integration testing. The ALHAT range, accuracy, and precision requirements for HDA and HRN push the performance limits of the current flash LIDAR camera (that uses components from the mid-2000's), but considerable enhancements have been made since the last ALHAT field test towards meeting these requirements for the testing planned this year. Figure 2 shows the current implementation of the three sensor subsystems.

\section{VTB System Implementation}

In the time since the last ALHAT field test 4 in 2010, each subsystem has gone through a cycle of re-design, repackaging and performance refinement to produce an ALHAT system suitable for integration with a VTB. In addition to mass, volume, and power reductions, modifications for thermal management, vibration isolation, and performance improvements from lessons learned during field testing have been incorporated. Figure 3 shows the ALHAT implementation for Field Test 4 together with a photo of the current configuration integrated with Morpheus. Field Test 4 used laboratory rackmounted equipment that required a large shipping container to house all the components. The current configuration has a total weight of approximately $160 \mathrm{~kg}$, and can be accommodated primarily on the Morpheus upper deck as shown in the figure.

Despite this substantial reduction in the system's mass and volume requirements, another

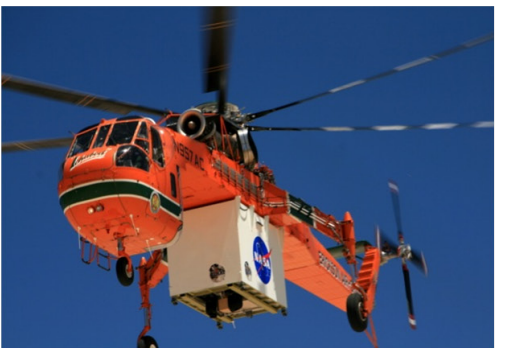

(A)

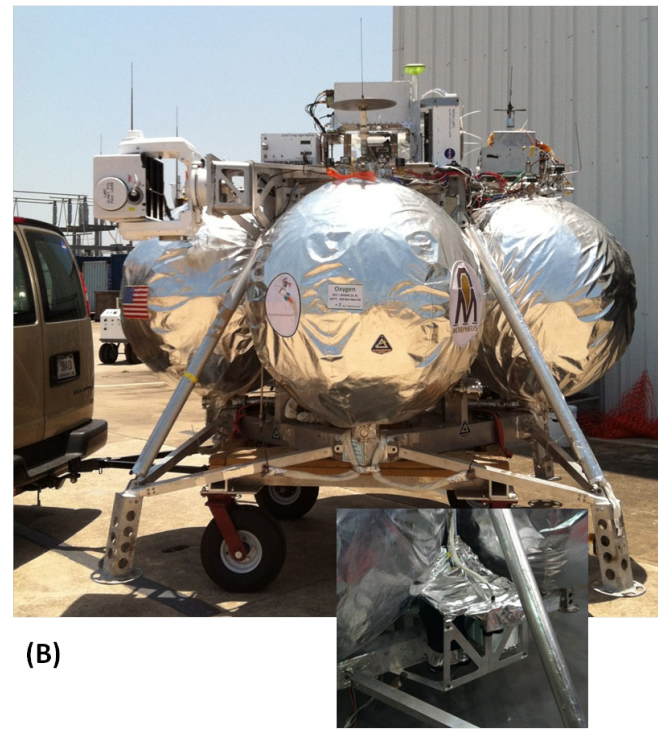

Figure 3. ALHAT test configurations: (A) Field Test 4 helicopter testbed system and (B) current VTB system.

4

American Institute of Aeronautics and Astronautics 
development cycle is required to make further mass and volume reductions for the system to be suitable for a space mission. Note that the current implementation was built with many Commercial Off The Shelf (COTS) and Modified Off The Shelf (MOTS) components in order to meet modest budget and aggressive schedule constraints. Section V provides more details about the work planned for the next ALHAT implementation.

Figure 4 is a block diagram showing the ALHAT system components and interfaces with the Morpheus vehicle. The color coding of each component in the figure highlights the cross-organizational nature of the ALHAT project. As shown in the figure, the ALHAT system interfaces with the Morpheus Avionics and Power Unit (APU), comprised of the vehicle flight computer and power distribution system. The ALHAT Doppler LIDAR and Laser Altimeter are powered from the vehicle. The Hazard Detection System (HDS) has its own flight batteries and power distribution system. The Morpheus Guidance,

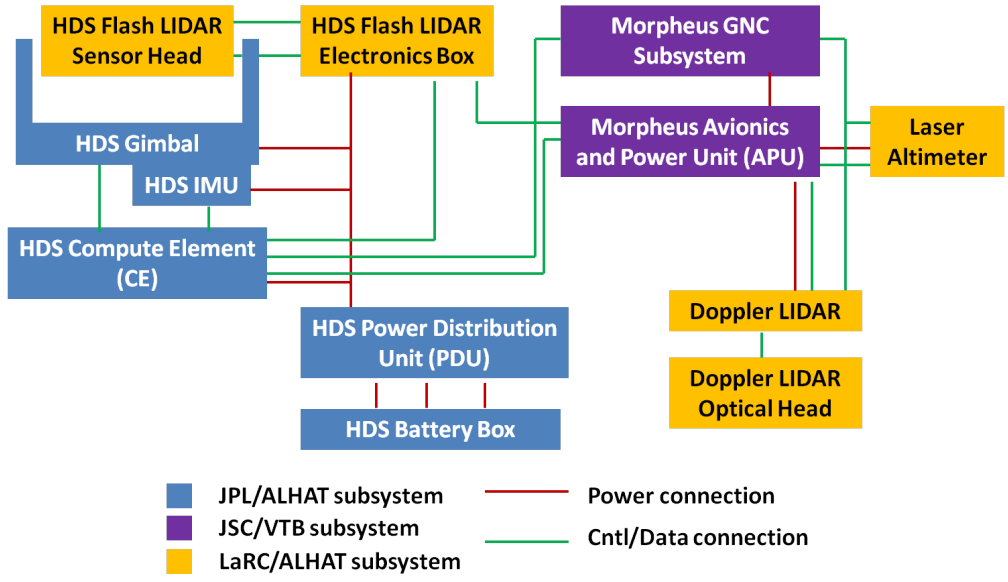

Figure 4. ALHAT/Morpheus interface block diagram.

Navigation, and Control (GN\&C) subsystem includes an Inertial Measurement Unit (IMU) and GPS receiver, and provides a Pulse Per Second (PPS) synchronization signal to the ALHAT subsystems that supply data to the vehicle's navigation filter. The HDS has its own IMU attached to the gimbal mounting plate to allow for vehicle motion compensation to be applied to the gimbal motion commands. The Morpheus APU performs the autonomous flight management and sends mode commands to the ALHAT system. In HDA mode, the HDS scans an area about the ILP, capturing a mosaic of flash LIDAR images. A Digital Elevation Map (DEM) is produced from this mosaic, and the hazard detection algorithm is executed to identify safe sites. A prioritized list of safe sites is provided to the host vehicle flight manager. ALHAT then transitions to HRN mode where surface features in successive DEMs are tracked and used to provide precision local navigation updates to the vehicle GN\&C system. Morpheus uses a dual navigation filter that simultaneously processes a solution using only the vehicle navigation sensors, and a solution that incorporates ALHAT navigation inputs. With this architecture the ALHAT navigation solution can be tested open loop from the vehicle guidance, and the navigation data source can be switched real-time if needed in closed loop testing.

\section{Integration and Test Activities in GFY2012}

To meet the ALHAT and Morpheus project goals for GFY2012, aggressive test campaigns were planned and executed. Both projects had a considerable amount of development and testing to perform prior to attempting to integrate ALHAT with the VTB. Availability of both test articles and key personnel for the two projects with ongoing internal development and test milestones was a constant challenge. The use of both standalone tests and integrated tests of opportunity was maximized. In order to accomplish the internal integration of the ALHAT subsystems that were literally being developed on opposite coasts of the United States, and to find as many issues as possible prior to attempting VTB integration, an ALHAT ground test campaign was performed at NASA Langley Research Center (LaRC). After these ground tests were completed, the ALHAT system was shipped to the Johnson Space Center (JSC) for integration with the Morpheus vehicle. At JSC, various static and dynamic tests on the vehicle were performed, concluding with a series of tethered flight tests. Finally, autonomous navigation, both open loop and closed loop with the ALHAT system, is planned during free flight tests at Kennedy Space Center (KSC). Each of these test phases is described in this section. 


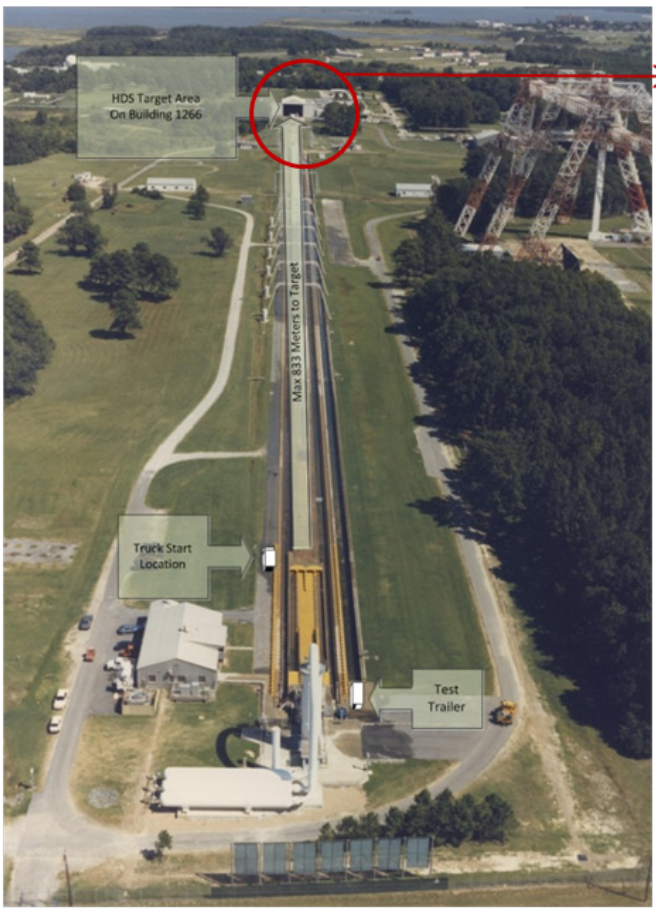

(A)

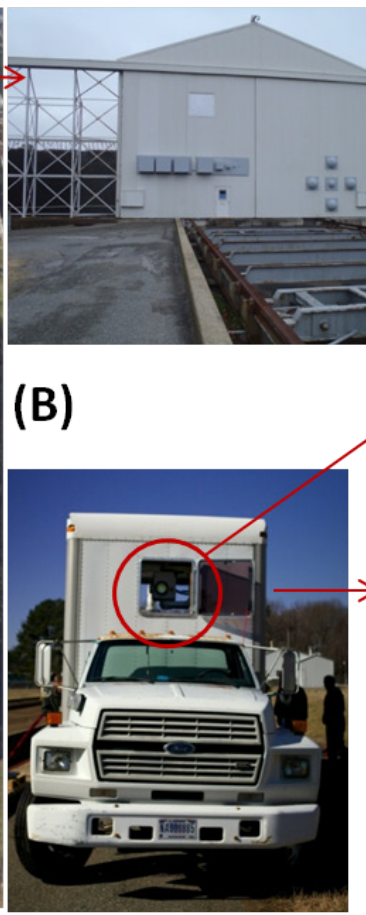

(C)

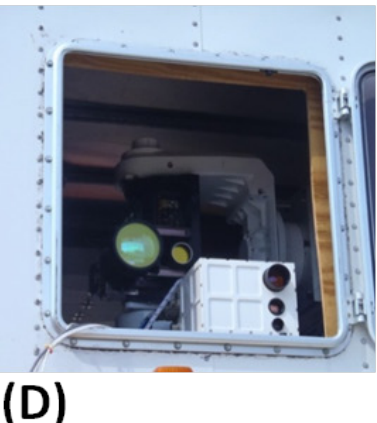

(D)

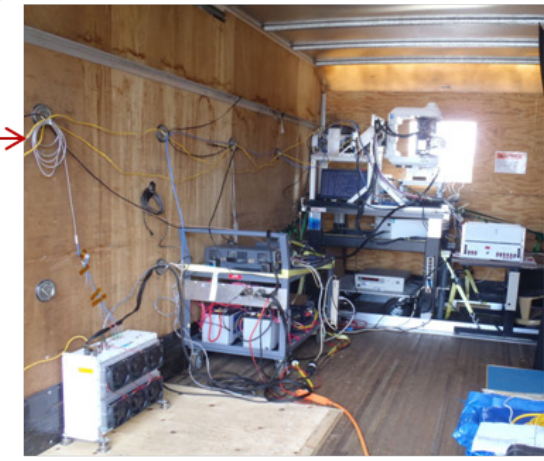

(E)

Figure 5. ALHAT dynamic ground test range configuration at the NASA Langley Long Distance Test Range (LDTR): (A) overview of range, (B) ALHAT LDTR target area, (C) truck host vehicle surrogate, (D) truck sensor window showing ALHAT flash LIDAR and laser altimeter, and (E) ALHAT system on truck.

\section{A. Ground Integration Tests}

Integrated system testing on a dynamic (truck) platform was conducted at LaRC Long Distance Test Range (LDTR). This facility was previously used for testing Space Shuttle tires on various simulated runway conditions, and features a long straight track where a steam-powered sled was used to generate Shuttle landing speeds. There is a road along one side of the sled track which ends at a shed that was used to house the sled. For ALHAT testing, the system was integrated with a truck that has a window in the front of the cargo area for sensor access. Targets were attached to the shed for imaging, and the truck was driven towards the targets while the ALHAT system operated. The test configuration is shown in Fig. 5. Using this approach, dynamic testing at distances similar to the planned VTB trajectory was accomplished. Morpheus components were not available at the time of the initial LaRC tests, so as a surrogate for the VTB the Charles Stark Draper Laboratory Guidance Embedded Navigator Integration Environment (GENIE) system was integrated on the truck with the ALHAT system ${ }^{5}$. This also served as integration testing for the ALHAT subsystems with the GENIE, which implements ALHAT autonomous flight management, guidance, and control, and is being tested on a commercial VTB. 


\section{B. VTB Range and Tether Tests}

Initial ALHAT/Morpheus integrated testing was performed in the Morpheus hangar at JSC, but this facility does not provide the distances required for focused flash LIDAR images. Testing with image data was performed on JSC's antenna test range, which is also the location for Morpheus tether testing. The ALHAT flash LIDAR is not eye-safe, and the antenna range allows for firing the LIDAR away from populated areas on the facility at target ranges of interest without laser hazard outside the perimeter of the facility. The objectives of the range testing include a verification of the static and dynamic end-to-end pointing accuracy of the HDS using the Morpheus navigation inputs (critical to HRN), the execution of a static and dynamic mosaic scan (critical to HDA), verification of the Morpheus navigation solution, and initial testing of the navigation sensors (Doppler LIDAR and Laser

(Not To Scale)

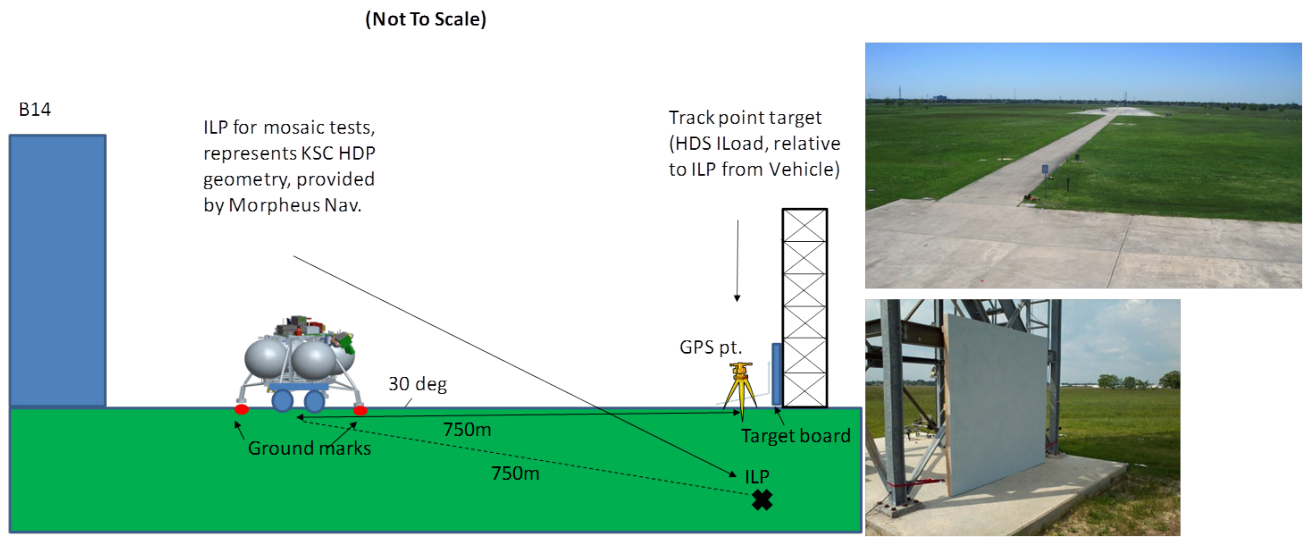

Figure 6. ALHAT/Morpheus range test configuration at NASA JSC.

Altimeter) in the flight environment. The goal was to expose as many issues in the range test configuration as possible, as a tether test configuration involves substantially more overhead. Figure 6 shows a schematic of the configuration for these tests and pictures of the range. Another test of opportunity in the range configuration was a lift test to collect Doppler LIDAR and laser altimeter data while the vehicle was raised and lowered on its tether crane. Figure 7 (A) is a photograph from this test. The tether testing objectives were the same as the range tests, with a similar configuration except the pointing target was placed about $340 \mathrm{~m}$ from the tether pad due to limited range space in the direction safe to fire the LIDAR. The track-pointing and mosaic functions were tested during a twolevel hover that provided altitude cues to the AFM to command the ALHAT HDS to change between track-ILP and mosaic operational modes. These tests were the first opportunity to test these operational modes in the flight

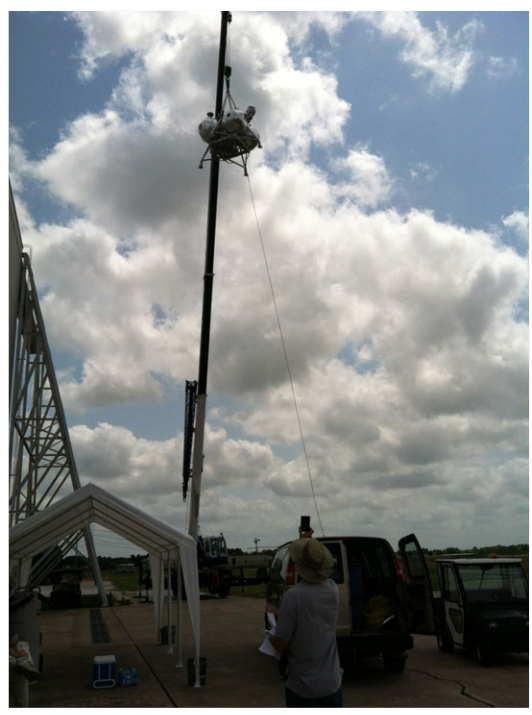

(A)

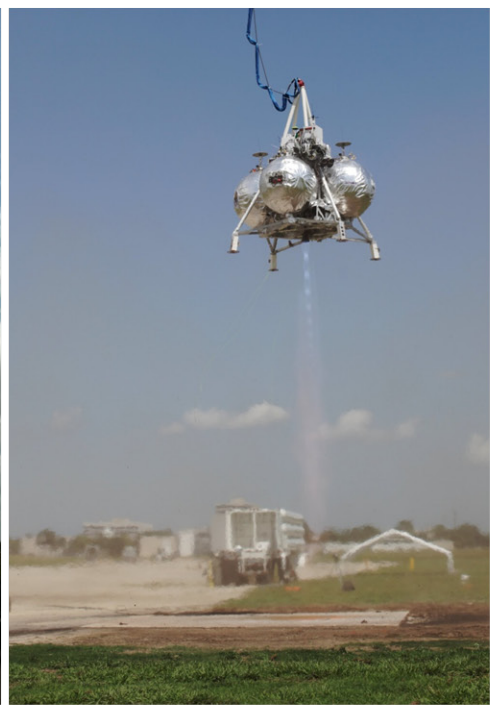

(B)

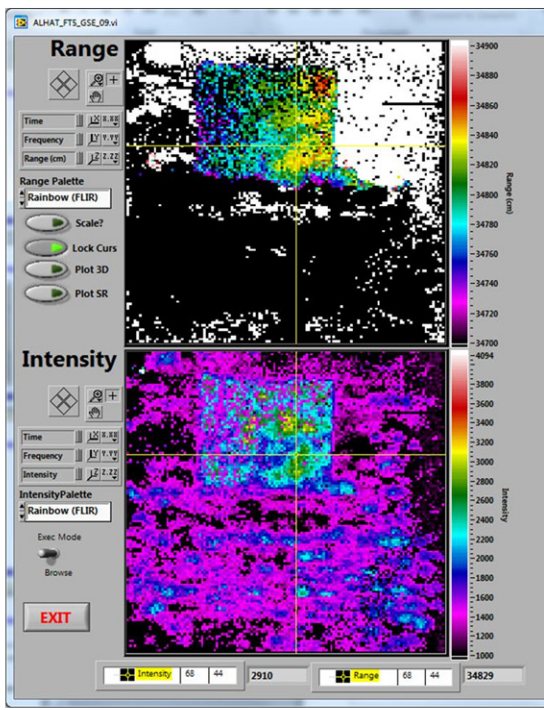

(C)

Figure 7. ALHAT/Morpheus testing at JSC: (A) lift test, (B) tether test, and (C) flash LIDAR image data of target board during tether pointing test. 
vibration environment. Figure 7 (B) shows Tether Test 18, and (C) shows the flash LIDAR range and intensity images of the target board during the flight. Although there was a large amount of pre-triggering of the flash LIDAR on dust raised from the engine, the results of these tests showed that the HDS pointing and mosaic execution functioned acceptably in the flight environment. The dust was an artifact of tether testing in close proximity to the ground, and will not be an issue in the planned KSC test trajectory. Additional benefits of this testing are that procedures, ground operator roles and display tools were matured to the level of supporting flight testing on the vehicle.

\section{VTB Free-Flight Tests}

The Shuttle Landing Facility (SLF) at NASA KSC was chosen as the location to perform freeflight testing of the Morpheus vehicle. The Morpheus test campaign at $\mathrm{KSC}$ is planned in four parts: 1) Demonstrate free-flight capability in short "hop" flights, 2) Perform envelope expansion of the free flight trajectory, 3) fly Hazard Detection Phase (HDP) free flights with the ALHAT system integrated and operating open loop with respect to the vehicle GN\&C, and

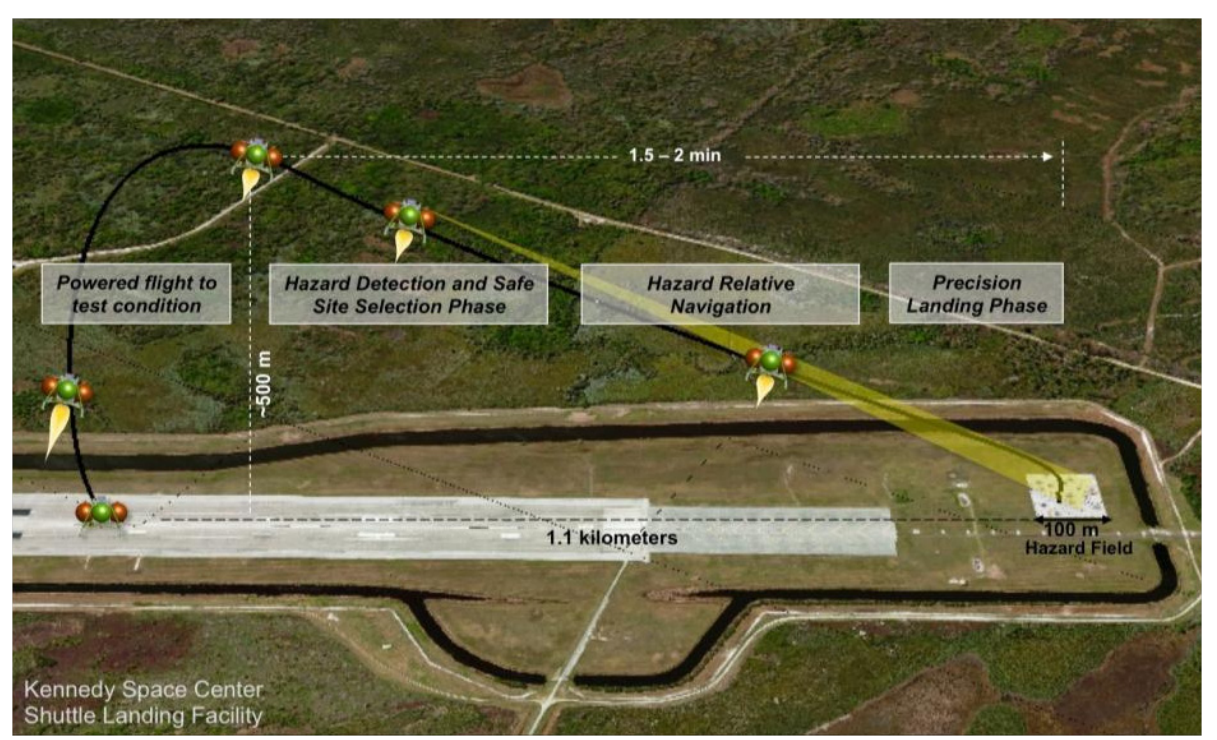

Figure 8. ALHAT/Morpheus HDP flight profile.

4) fly HDP trajectories with the ALHAT system operating closed loop with respect to the vehicle GN\&C. The HDP trajectory starts after the pitch-up maneuver and transitions the lander into a powered descent profile (see Fig. 1). Figure 8 shows a drawing of this trajectory at the SLF. In order to provide realistic terrain to scan and process for hazard detection and avoidance a $100 \mathrm{~m}$ x $100 \mathrm{~m}$ area was constructed to resemble an actual area of the Moon, with craters, slopes, and rocks. To accomplish this, NASA JSC provided the requirements to JPL, who then designed a DEM that meets those criteria. A build-to specification was created from the DEM, and NASA KSC constructed the field near the north end of the SLF runway. Figure 9 includes a photograph of the completed hazard field, as well as a plot of the model that was used as the basis for its design. A major concern was weathering of the field after completion, particularly with the frequent storms KSC experiences in the summer. KSC solved this problem by using a material called "crawler way fines" which is the pulverized stone that paved the Shuttle crawler road from the vehicle assembly building to the launch pads. These stones were shipped to KSC during the Shuttle Program because they had the right crush characteristics to minimize vibration on the Shuttle/external tank/SRB stack during transit to the launch pad. The stones are crushed after the passage of the Shuttle and have to be refreshed. KSC has an abundance of this material and realized that after it is wet down, it forms a hard crust resistant to weathering. Samples were sent to NASA Langley to evaluate reflectivity properties and it was determined that the material would be suitable to construct the hazard field.

\section{Lessons Learned}

The ambitious goals of this year pursued by the collaboration of the ALHAT and Morpheus projects generated many learning opportunities, and a few of interest are discussed in this section. 


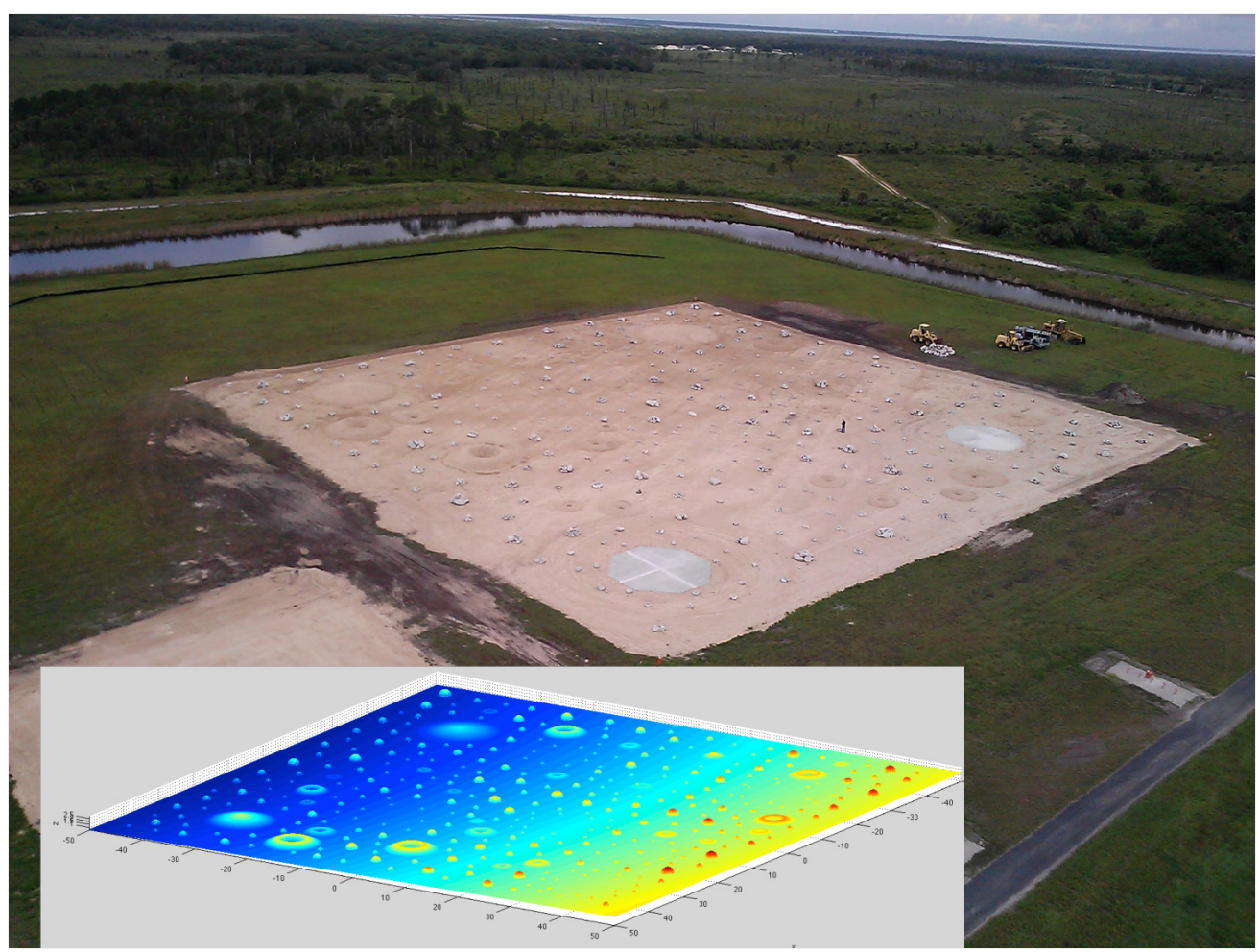

Figure 9. ALHAT hazard field constructed at NASA KSC's SLF. Inset is a digital elevation map of the design JPL provided KSC to build the field.

- Create and push for early integration opportunities. As previously mentioned, the ALHAT system is developed by several NASA centers and partner organizations, separated geographically across the United States. Bringing the subsystems together for integrated ground testing exposed problems that could only be found in that configuration, and significantly reduced the schedule risk for Morpheus integration. In addition, the integration schedule created the "line in the sand" that drove the subsystem development, interface definition, and documentation, and enabled the maturity of these products to be assessed at an intermediate milestone. As a specific example of prioritizing early integration tests, the Morpheus project did not have an additional APU to be spared for ALHAT ground testing. As discussed in Section IV, ALHAT used the GENIE system as a navigation base and flight computer during ground tests at LaRC. To check interfaces and commanding with Morpheus, a partial set of APU components was sent to LaRC and tested with ALHAT. The partial APU was not able to support dynamic truck testing, but did allow the verification of the ALHAT command and data interfaces with the vehicle.

- Strive for fidelity in testing prior to the final system configuration. As shown in the example of using a subset of APU components, early integration often involves testing with incomplete configurations, or surrogates for certain systems not available at that stage of development. ALHAT provided mass simulators to the Morpheus project for early testing, and due to various resource constraints the simulators for different components had different levels of fidelity. The simulators that did not provide the correct volume properties contributed to some unexpected interference issues found in the ALHAT mechanical integration with Morpheus. These issues arose despite CAD models being developed for the vehicle with the ALHAT components installed. Another example is an issue discovered during integrated tests with Morpheus not found in dynamic ground testing. Although an effort was made to design the Morpheus and GENIE interfaces to be similar, a timing discrepancy between the Morpheus navigation state data and the ALHAT HDS navigation data was discovered that did not occur in tests using the GENIE system.

- Watch system modifications closely during a test campaign. An analog to fidelity in test configurations is keeping configurations as stable as possible through various stages of testing. Ideally, a system is not modified through testing and flight. But a reasonable balance must be determined in a technology 
demonstration project, in which a prototype system may still be developing towards its flight configuration, and issues are discovered during testing that must be addressed with modifications. For example, the flash LIDAR was found to operate close to its thermal limits in the ground integration tests, so more fans were added to the sensor head. This of course changed this subsystem's power consumption, and it was discovered during initial tests on Morpheus that the flash LIDAR flight battery provided very little margin over the expected operational time of the LIDAR. Batteries were added to the circuit, resulting in mass growth of the ALHAT system.

These lessons learned can be summarized as test early and often, test as you fly, and fly as you test. These tenants are not new, but the practical challenges of following them are shared here as similar experiences likely exist in other complex projects.

\section{Conclusion and Future Direction}

During Field Test 3 on a B-200 airplane and Field Test 4 on the Erickson Air-Crane helicopter, the ALHAT system included several large, heavy racks of avionics hardware for sensor command, control, and data processing. An onboard operator also enabled manual command and control of the gimbal and sensors. Over the past two years, the ALHAT Project has focused on the refinement and integration of the hazard detection and navigation sensors and avionics from Field Test 4 . The resulting GFY12 ALHAT prototype system is significantly more compact, robust, and automated than its Field Test 4 predecessor. The upcoming flight campaign at KSC will mark the first time that autonomous hazard detection and precision landing has been demonstrated during a high-energy landing trajectory on a rocket-powered testbed, and will represent a major step towards enabling safe, precision landing for future planetary exploration missions. The mass properties for the current ALHAT system are provided in Table 1. Approximately $95 \%$ of the total system mass is documented from measurements of the prototype hardware. Based upon the experience gained during the GFY11 to GFY12 development cycle, the ALHAT team has developed a plan to incrementally reduce the Size, Weight, and Power ( $\mathrm{SWaP}$ ) of the ALHAT system via a series of design refinements, leading towards a more spaceflight-capable ALHAT system that could be ready to test on a VTB in

Table 1. ALHAT system current and projected mass properties.

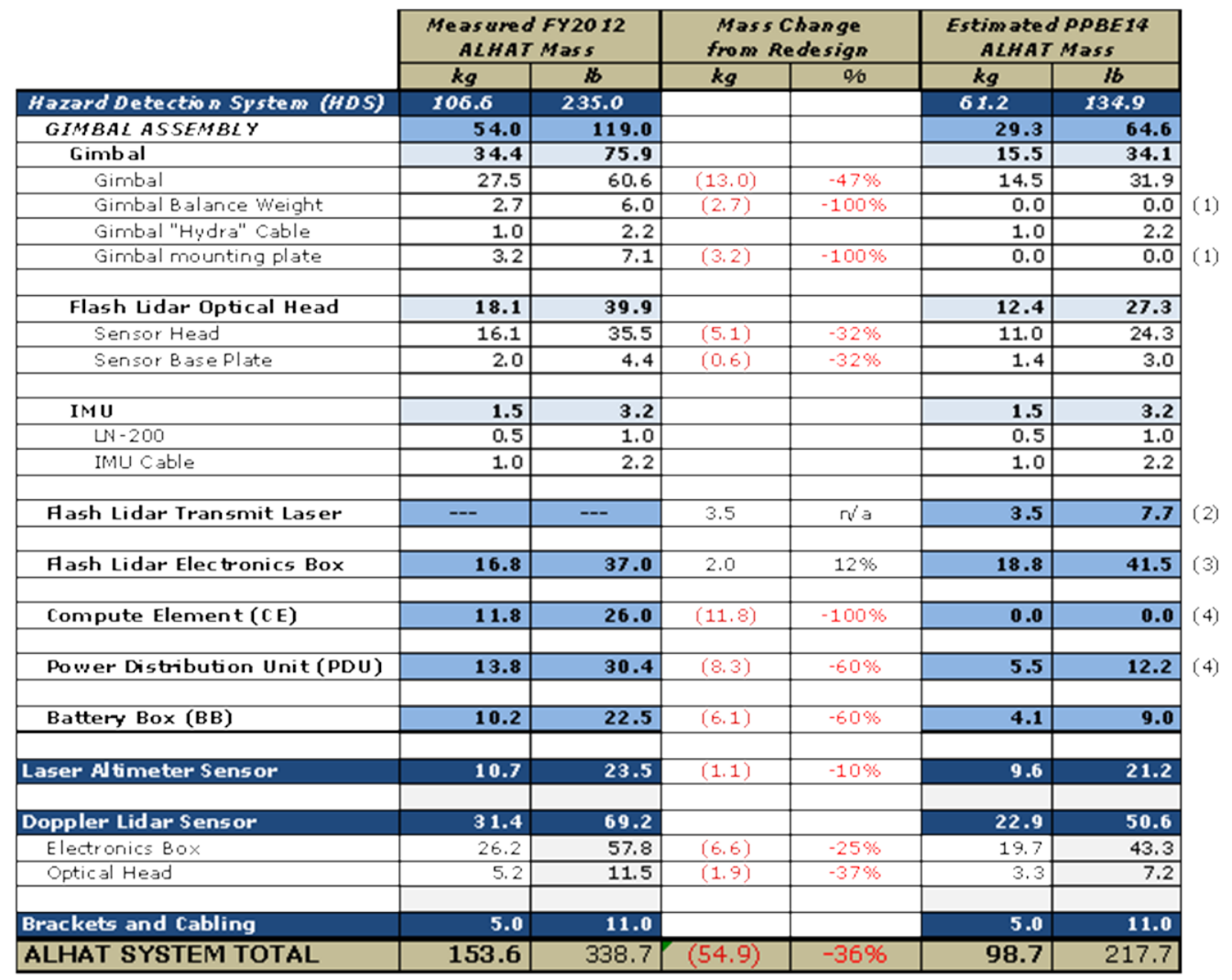


GFY14. Referencing the numbers along the right size of Table 1, refinement (1) saves nearly $6 \mathrm{~kg}$ by the elimination of the separate gimbal mounting plate and the gimbal balance weight. The gimbal and flash LIDAR optical head would be redesigned for balance without a large trim weight, and the gimbal would be directly attached to the host vehicle structure. Refinement (2) uses an advanced, high-power fiber optic cable developed by Goddard Space Flight Center (GSFC) to move the flash LIDAR laser from the gimbaled optical head to a fixed location on the host vehicle. This configuration change reduces the size of the gimbal as well as the articulated mass of the sensor, and also simplifies thermal control for the laser. Refinement (3) replaces the entire HDS Compute Element with a singleboard computer hosting the Maestro multi-core processor and a Field Programmable Gate Array (FPGA). The Maestro processor incorporates native floating-point capability and was developed for the space environment from the Tilera Tile64 processor using Radiation-Hardened By Design (RHBD) techniques. The Maestro board would be integrated with an existing avionics chassis, possibly even serving as the host vehicle flight computer. Refinement (4) represents an estimate of the mass savings that could be achieved by integrating the HDS power system with the host vehicle and optimizing the HDS to reduce power consumption. As shown, an overall mass saving of one-third or greater can be achieved over the next two years through incremental ALHAT design improvements and tighter Morpheus integration.

The ALHAT team is studying strategies for even more substantial improvements in system SWaP, including new active LIDAR sensor and beam steering technologies that have the potential to greatly reduce laser power and eliminate the gimbal. In addition, custom PC boards could be implemented to reduce the number of separate processor and I/O cards and consolidate HDS functions. These custom PC boards would also be designed with greater emphasis on efficient thermal control to reduce the weight and power required for the fans and thermoelectric coolers (TECs) currently embedded within the ALHAT components. An emphasis on conductive cooling would further improve thermal integration with the Morpheus VTB and provide a more spaceflight-like component configuration in support of future spaceflight missions. This next-generation ALHAT system would likely have a total mass of less than $75 \mathrm{~kg}$, and would facilitate the infusion of ALHAT technologies into a future planetary exploration mission.

\section{Acknowledgments}

The work contained in this paper is gathered from the efforts of the ALHAT and Morpheus project teams and is in no way done solely by the authors. The authors wish to thank the ALHAT and Morpheus teams specifically for their outstanding efforts supporting the aggressive goals of this year. The authors also wish to thank the team at Kennedy Space Center for their outstanding support of the free flight test activities.

\section{References}

\footnotetext{
${ }^{1}$ Brady, T. and Paschall, S., "The Challenge of Safe Lunar Landing”, Aerospace Conference, 2010 IEEE, March 2010, pp. 1-14.

${ }^{2}$ Epp, C., Robertson, E., and Brady, T., "Autonomous Landing and Hazard Avoidance Technology (ALHAT)”, Aerospace Conference, 2008 IEEE, March 2008, pp. 1-7.

${ }^{3}$ Striepe, S., Robertson, E., and Epp, C., "Autonomous Precision Landing and Hazard Avoidance Technology (ALHAT) Project Status as of May 2010", International Planetary Probe Workshop 2010, Barcelona, Spain, June 2010.

${ }^{4}$ Brady, T., Bailey, E., Crain, T., and Paschall, S., “ALHAT System Validation”, $8^{\text {th }}$ International ESA Conference on Guidance, Navigation \& Control Systems, Karlovy Vary, Czech Republic, June 2011.

${ }^{5}$ Paschall, S. and Brady, T., "Demonstration of a Safe \& Precise Planetary Landing System On-Board a Terrestrial Rocket," Aerospace Conference, 2012 IEEE, March 2012, pp. 1-8.

${ }^{6}$ Larson, W., Kirkpatrick, D., Sellers, J., Thomas, D., and Verma, D., Applied Space Systems Engineering, McGraw-Hill, Boston, 2009, p. 575.

${ }^{7}$ Project Morpheus Website, URL: http://morpheuslander.jsc.nasa.gov/ [cited 24 July 2012].

${ }^{8}$ NASA Office of the Chief Technologist, "Space Technology Roadmaps, the Future Brought to You by NASA", URL: http://www.nasa.gov/offices/oct/home/roadmaps/index.html [cited 24 July 2012].

9 Catenaro, R., "Preliminary Report on Automatic LEM Mission Study", Grumman Aircraft Engineering Corporation, Rept. LED-54-02, April 29, 1963. URL: http://ntrs.nasa.gov [cited 30 July 2012].

${ }^{10}$ Hudson, W. and Johnston, G., "Spacecraft Technology Requirements for Future NASA Missions", AIAA Space Programs and Technologies Conference, AIAA, Washington, D.C., 1988, pp. 1-9.

${ }^{11}$ Pien, H., "Autonomous Hazard Detection And Avoidance", NASA CR-188331, 1992.

12 Epp, C.D. and Smith, T.B., "Autonomous Precision Landing and Hazard Detection and Avoidance Technology (ALHAT)," Aerospace Conference, 2007 IEEE, March 2007, pp. 1-7.
} 
${ }^{13}$ Fisackerly, R. et al.,"The ESA Lunar Lander Mission", AIAA SPACE 2011 Conference and Exposition, Long Beach, California, Sep., 2011.

14 Allen, Andrew, C. M., "Full-Scale Testing and Platform Stabilization of a Scanning Lidar System for Planetary Landing", Proceedings of SPIE, Volume 6960, Editor, Wolfgang Fink, Bellingham, Washington, May 2008.

${ }^{15}$ Mahmood, W., Shah, S., "Vision Based Hazard Detection and Obstacle Avoidance for Planetary Landing," Nonlinear Dynamics and Synchronization, 2009. INDS '09. 2nd International Workshop on, July 2009, pp. 175-181.

${ }^{16}$ Feng J., Cui P., Cui H., "Autonomous Hazard Detection and Landing Point Selecting for Planetary Landing," Systems and Control in Aeronautics and Astronautics (ISSCAA), 2010 3rd International Symposium on, June 2010, pp. 1292-1296.

${ }^{17}$ Rohrschneider, R.R., and Coppock, E., "Performance of a Real-Time Filter-Based Hazard Detection Algorithm," Aerospace Conference, 2011 IEEE, March 2011, pp. 1-10.

${ }^{18}$ Chakroborty, S., Aboutalib, O., and Meade, C., "Autonomous Lunar Lander Hazard Detection and Avoidance System", AIAA SPACE 2009 Conference and Exposition, Pasadena, California, Sep. 2009.

${ }^{19}$ Olansen, J. B., Munday, S. R., Mitchell, J. D., and Baine, M., "Morpheus: Advancing Technologies for Human Exploration”, Global Exploration Conference 2012, May 2012, Washington, DC.

${ }^{20}$ Villalpando, C., Johnson, A., Some, R., Oberlin, J., and Goldberg, S., "Investigation of the Tilera Processor For Real Time Hazard Detection and Avoidance on the Altair Lunar Lander," Aerospace Conference, 2010 IEEE, March, 2010, pp. 1-9.

${ }^{21}$ Huertas, A., Johnson, A.E., Werner, R.A., and Maddock, R.A., "Performance Evaluation of Hazard Detection and Avoidance Algorithms For Safe Lunar Landings," Aerospace Conference, 2010 IEEE, March 2010, pp.1-20.

22 Amzajerdian, F., et al., "Doppler Lidar Sensor For Precision Landing on the Moon and Mars," Aerospace Conference, 2012 IEEE, March 2012, pp.1-7.

${ }^{23}$ Amzajerdian, F., Pierrottet, D., Petway, L., and Vanek, M., "Development of LIDAR Sensor Systems For Autonomous Safe Landing on Planetary Bodies", International Conference on Space Optics, Rhodes, Greece, October 2010. 